\title{
Intensified chemotherapy and simultaneous treatment with heparin in outpatients with pancreatic cancer - the CONKO 004 pilot trial
}

Uwe Pelzer $^{1 *}$, Andreas Hilbig ${ }^{1,2}$, Jens M Stieler ${ }^{1}$, Marcus Bahra ${ }^{3}$, Marianne Sinn ${ }^{1}$, Bernhard Gebauer ${ }^{4}$, Bernd Dörken ${ }^{1}$ and Hanno Riess ${ }^{1}$

\begin{abstract}
Background: Advanced pancreatic cancer (APC), beside its high mortality, causes the highest rates of venous thromboembolic events (VTE). Enoxaparin, a low molecular weight heparin (LMWH), is effective in prevention and treatment of VTE. Some small studies indicated that this benefit might extend to patients with cancer and probably prolong survival due to independent mechanisms. We initiated this safety investigation to get feasibility information on intensified chemotherapy combined with LMWH in outpatients with APC treated in $1^{\text {st }}$ line.

Methods: The trial was a prospective, open-label, single center investigation in outpatients with inoperable pancreatic cancer who were treated with intensified first-line chemotherapy along with concomitant application of subcutaneous LMWH. The combined chemotherapy consisted of gemcitabine $1 \mathrm{~g} / \mathrm{m}^{2}$ (30 min), 5-FU $750 \mathrm{mg} / \mathrm{m}^{2}$ (24 h), folinic acid $200 \mathrm{mg} / \mathrm{m}^{2}$ (30 min), and Cisplatin $30 \mathrm{mg} / \mathrm{m}^{2}$ (90 min) on day 1 and 8; q3w for the first 12 weeks (GFFC) followed by gemcitabine alone in patients without cancer progression. The simultaneous application of prophylactic enoxaparin started on day 1 of chemotherapy with a fixed dose of $40 \mathrm{mg}$ daily. Statistical analyses were performed using R 3.01 with software package CMPRSK and SPSS software v19.0.
\end{abstract}

Results: The investigation was stopped after recruitment of 19 patients. At this time 15 patients had completed the required 12 weeks of treatment. Based on 71 cycles of GFFC + enoxaparin (median 4/pt [range: 2-4]) and 108 cycles of single-agent gemcitabine + enoxaparin (median 4/pt [range: 0-18]) the cumulative frequency of NCI-CTC toxicities grade $3 / 4$ was below $10 \%$. One case (5\%) of a symptomatic non-lethal thromboembolic event was observed while receiving LMWH treatment. No severe bleeding event as defined in the protocol has been observed. The median overall survival was 10.05 [95\% Cl: 8.67-18.14] months.

Conclusions: The addition of enoxaparin to GFFC chemotherapy is feasible, safe and does not appear to affect the efficacy or the toxicity profile of the chemotherapy regimen in patients with advanced pancreatic adenocarcinoma. Based on these findings we have initiated the randomized CONKO-004 trial to examine whether enoxaparin reduces the incidence of thromboembolic events or increases overall outcome.

Trial registration: Clinical Trials NCT01945879.

\footnotetext{
* Correspondence: uwe.pelzer@charite.de

'Department of Hematology/Oncology, CharitéCentrum für Tumormedizin, Charité - Universitätsmedizin Berlin, Augustenburger Platz 1, Berlin 13353, Germany

Full list of author information is available at the end of the article
}

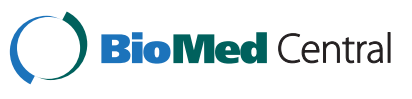

(c) 2014 Pelzer et al.; licensee BioMed Central Ltd. This is an Open Access article distributed under the terms of the Creative Commons Attribution License (http://creativecommons.org/licenses/by/2.0), which permits unrestricted use, distribution, and reproduction in any medium, provided the original work is properly credited. The Creative Commons Public Domain Dedication waiver (http://creativecommons.org/publicdomain/zero/1.0/) applies to the data made available in this article, unless otherwise stated. 


\section{Background}

Pancreatic adenocarcinoma is an aggressive cancer type with early extensive local invasion, rapid systemic spread combined with a high resistance to chemotherapy. This is accompanied by a case fatality rate of $90 \%$ and thus constitutes the fourth most-frequent cause of death from solid cancer in the western world [1]. In patients with non-resectable advanced pancreatic cancer (APC) median overall survival without effective systemic anticancer treatment is not higher than 4 months [2-4]. Despite intensive anticancer research in the last decade, five year overall survival in patients with APC is still less than 5\% [5]. Single agent gemcitabine (GEM) has become the standard first-line chemotherapy for pancreatic cancer 15 years ago [2]. More recent trials using combination regimens with or without gemcitabine showed improvements in cancer control-rate and even survival advantages, most pronounced for patients with better performance status [6-9]. Thus, patients with good performance status are indicated for intensified treatment with combined chemotherapy regimens $[9,10]$.

Venous thromboembolic events (VTE) are considered to be a commonly accurring major lethal complication in cancer patients. Population based case-control trials display a cumulative incidence of VTE of up to six-fold in cancer patients [11]. Cancer types with highest incidence rates are advanced malignancies of the brain, pancreas, lung, and stomach [12-14]. As compared to more limited stages metastatic disease results in a 4 to 13 -fold elevated VTE risk, which is further increased by systemic anticancer therapy [14]. The bidirectional interaction between cancer and hemostasis not only leads to activation of blood cells and the coagulation system resulting in clinically relevant thromboembolism but these processes are also under suspicion to enhance cancer growth and metastatic spread [15]. VTE are considered to be a prognostic-negative factor $[11,12]$ and small studies showed astonishing survival advantages using heparin as prophylactic treatment to prevent VTE [16]. Based on these assumptions our CONKO study group planned to conduct a randomized trial to investigate the impact of low molecular weight heparin (LMWH) in a prospective setting in patients with advanced pancreatic cancer undergoing first line therapy, the CONKO-004 trial [17]. During the preliminary stages we had to undertake a pilot trial to get information on safety and feasibility of combined chemotherapy with simultaneous treatment of the LMWH enoxaparin in patients with advanced pancreatic cancer who are at high risk of gastrointestinal bleeding due to local cancer spread. The toxicity profile, feasibility, and maximum tolerable dosage of the combination of gemcitabine/5-FU/Folinic Acid/Cisplatin was investigated in a past phase-I trial of our study group [18].

\section{Methods}

\section{Design and treatment}

The trial was a prospective, open-label, single center investigation in patients with advanced pancreatic cancer who were treated with first-line chemotherapy in an outpatient setting. The intensified treatment consisted of gemcitabine $1 \mathrm{~g} / \mathrm{m}^{2}$ (30 $\left.\mathrm{min}\right), 5$-FU $750 \mathrm{mg} / \mathrm{m}^{2}$ (24 h), folinic acid $200 \mathrm{mg} / \mathrm{m}^{2}$ (30 min), and cisplatin $30 \mathrm{mg} / \mathrm{m}^{2}$ (90 min) on day 1 and 8; q3w. The concomitant application of enoxaparin started on day 1 of chemotherapy with a fixed dose of $40 \mathrm{mg}$ daily until cancer progression. Beyond the initial 3 months of intensified $1^{\text {st }}$-line chemotherapy all patients without cancer progression received further treatment with single agent gemcitabine and enoxaparin to prevent patients from cumulative toxicities. Dose adjustment for enoxaparin was recommended in patients with impaired kidney function or thrombocytopenia within the study according to NCI-CTC (National Cancer Institute Common Toxicity Criteria) guidelines to minimize bleeding risk. Dose adjustment for chemotherapy dosage was realised by protocol-defined regulations [18]. Prophylactic antiemetic therapy and supportive care were provided according to individual symptoms and demand.

The study used a sequential design to be able to stop the feasibility investigation in case of severe side effects. After inclusion of three consecutive patients a hold of recruitment was arranged until all three patients received at least 4 weeks of concomitant enoxaparin treatment. In absence of inacceptable toxicity by confirmation of the protocol committee the recruitment was continued until a minimum of 15 patients received at least 12 weeks concomitant enoxaparin treatment. Patients were followed up until death of any reason or lost to follow-up.

The trial was approved by the scientific and research ethics committee of our institution (Ethikkommission der Charité - Universitaetsmedizin Berlin). The investigation was conducted in accordance with the Declaration of Helsinki and Good Clinical Practice Guidelines and within the CONSORT guidelines. Furthermore, the national principles for the proper execution of the clinical examination of drugs (Bundesanzeiger No. 243 of 30.12.1987), the national regulations of the German drug law, and the German drug test guidelines were adhered. Trial registration: Clinical Trials NCT01945879.

\section{Eligibility criteria}

Main inclusion criteria were: ambulatory patients with histologically confirmed APC, no previous radio- or chemotherapy, Karnofsky Performance Status (KPS) $\geq 60 \%$; measurable tumour lesion confirmed by computed tomography (CT) or magnetic resonance imaging (MRI) within the last 14 days, no VTE within the last 2 years, adequate compliance and home residence within geographical proximity to our department (allowing an adequate follow-up), 
sufficient bone marrow function (leukocytes $3.5 \times 10^{9} / \mathrm{l}$, thrombocytes $\left.100 \times 10^{9} / 1\right)$, age $\geq 18$ years.

Patients were excluded in case of pre-existing indication for anticoagulation, major bleeding events within the last 2 weeks or severe impairment of coagulation, active gastrointestinal ulcers or major surgery within the last 2 weeks, body weight $<45 \mathrm{~kg}$ or $>100 \mathrm{~kg}$, pregnancy/lactation or insufficient contraception during study or severely impaired renal function (creatinine clearance $<30 \mathrm{ml} / \mathrm{min}$ ).

All patients had to provide written informed consent.

\section{Outcome measures}

Primary investigation included the feasibility, the toxicity profile, and probable drug interactions. The first step was the analysis of safety data of the initial three patients after completing their first 4 weeks of combined treatment. On condition that there is no more than one patient with a toxicity of NCI-CTC grade $3 / 4$ and no event of severe bleeding, the recruitment would be continued (protocol committee consensus). The final treatment feasibility and safety would be approved if the incidence of predefined severe grade $3 / 4$ toxicities (NCI-CTC) would be no higher than $20 \%$ as well as the incidence of predefined severe, life threatening bleeding events would be no higher than $20 \%$ in a minimum of 15 patients under treatment duration of at least 12 weeks.

Secondary aims were overall survival (OS), influence of prognostic factors and the rate of symptomatic venous thromboembolic events and severe bleedings. Staging CT or MRI was performed at least every 12 weeks (earlier in case of suspected progression). According to safety aspects we documented severe bleedings in the event of short term decline of hemoglobin level $(\geq 2 \mathrm{~g} / \mathrm{dl} / 48 \mathrm{~h})$ in absence of other evidence (e.g. hemolysis) and/or the need for at least two red blood cell concentrates in case of confirmed blood loss and/or the clinical occurrence of serious apparent bleeding in parenchyma, muscle or cerebrum. The highest grade of a hematologically or nonhematologically toxicity during a cycle was recorded for the analysis.

\section{Statistical methods}

Statistical analyses were performed using R 3.01 with software package CMPRSK and SPSS software 19.0.

\section{Results}

For the first three patients no intense toxicity, particularly no severe bleeding event, was observed within the required 4 weeks of treatment. Hence the recruitment was continued to get a minimum of 15 patients receiving combined chemotherapy and concomitant enoxaparin over a duration of at least 12 weeks.

As 15 patients completed the 12 weeks of treatment a total number of 19 patients were recruited (Table 1). 2
Table 1 Demographic and baseline characteristics

\begin{tabular}{ll}
\hline Characteristic: $\mathbf{N}=\mathbf{1 9}(\mathbf{1 0 0} \%)$ & Chemotherapy + Enoxaparin \\
\hline Age - Years & $59[46-74]$ \\
Median [range] & \\
Sex - no. (\%) & $6(32)$ \\
Female & $13(68)$ \\
Male & \\
KPS - no. (\%) & $2(11)$ \\
$60 \%$ - 70\% & $17(89)$ \\
$80 \%$ - 100\% & \\
Stage - no. (\%) & $6(32)$ \\
M0 & $13(68)$ \\
M1 & $1(5)$ \\
Liver and lung & $8(41)$ \\
Liver & $2(11)$ \\
Lung & $2(11)$ \\
Other & $12(63)$ \\
Primary cancer - no. (\%) & $7(37)$ \\
Recurrent cancer - no. (\%) & \\
Site of recurrence & $5(71)$ \\
Liver & $2(37)$ \\
Local &
\end{tabular}

patients had progressive disease within the 12 weeks and decreased rapidly in performance status without qualifying for second line treatment. The remaining 17 patients were staged at 12 weeks, of which 15 patients had a stable disease and were getting further treatment with gemcitabine alone simultaneous with enoxaparin, whereas 2 patients had documented progressive disease and were switched to paclitaxel second-line regimen without enoxaparin treatment.

The analysis of cumulative toxicities was based on 71 (median 4/pt [range:2-4]) cycles of GFFC with enoxaparin and 108 (median 4/pt [range:0-18]) cycles of single-agent gemcitabine with enoxaparin (Figure 1). The incidence of NCI-CTC grade $3 / 4$ toxicities was below $10 \%$, most frequent side effect was neutropenia (grade 3: 12 cycles, grade 4: 2 cycles) and thrombocytopenia (grade 3: 1 cycle). During the course of GFFC the dose of cisplatin was reduced by $50 \%$ in 3 patients due to elevated serum creatinine levels, up to grade 2 in one patient. No grade $3 / 4$ nausea or vomiting occurred. One case of symptomatic deep venous thrombosis with consecutive non-lethal pulmonary embolism was observed while receiving prophylactic LMWH treatment (5\%). No patient stopped the subcutaneous heparin injections due to occurrence of local side irritations or generally inconvenience. No severe bleeding events as defined in the protocol were observed 


\begin{tabular}{|c|c|c|c|c|}
\hline & Grade I & Grade II & Grade III & Grade IV \\
\hline Anemia & 27 & 14 & 1 & \\
\hline Transaminases & 19 & & 1 & \\
\hline Nausea, Vomiting & 16 & 4 & & \\
\hline Neutropenia & 9 & 10 & 10 & 2 \\
\hline Creatinine & 7 & 1 & & \\
\hline Diarrhea & 5 & 3 & & \\
\hline Fever & 2 & 3 & & \\
\hline Thrombopenia & 1 & 2 & 1 & \\
\hline \multicolumn{5}{|l|}{ Bilirubin } \\
\hline \multicolumn{5}{|c|}{$\begin{array}{l}\text { Figure } 1 \text { Hematological and non-hematological toxicities } \\
\text { (no. out of } 179 \text { cycles). Numbers represent the count of } \\
\text { treatment cycles with corresponding toxicity. }\end{array}$} \\
\hline
\end{tabular}

under treatment with enoxaparin as well as in a corresponding follow-up of 30 days.

The median overall survival was 10.05 [95\% CI: 8.6718.14] months (see Figure 2). Two out of 19 patients were lost to follow-up and were censored at the time of last documented visit. The multivariate analysis of overall survival showed no significant effect on survival by a single characteristic (Figure 3). Best trend was observed

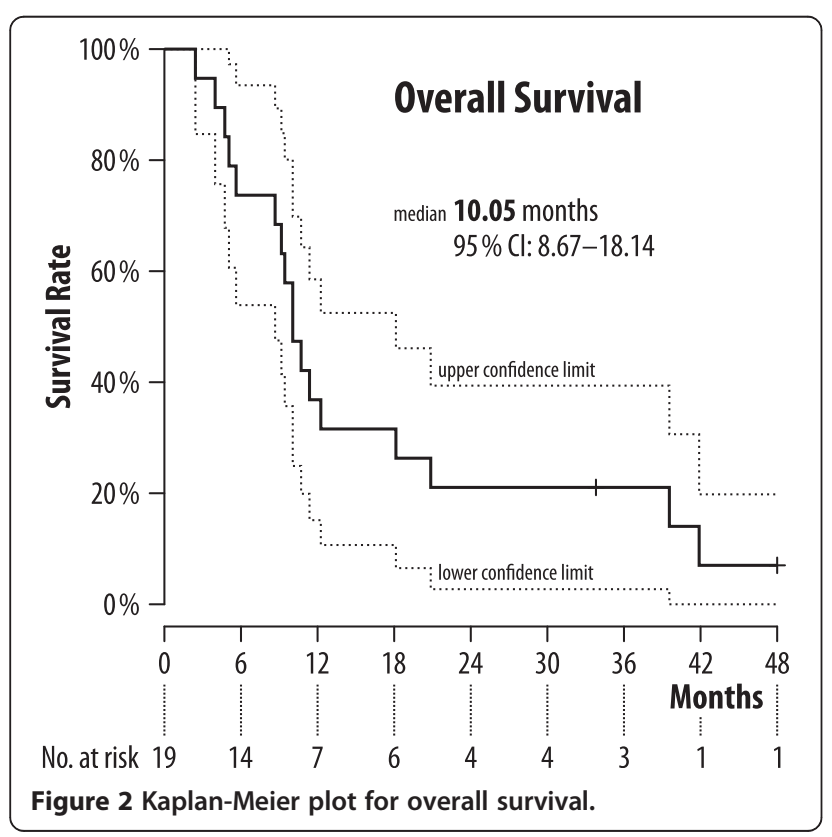

for patients with previous curatively intended cancer resection followed by cancer recurrence versus patients with primary inoperable pancreatic adenocarcinoma.

\section{Discussion}

This investigation was conducted to assess the use of intensified chemotherapy together with simultaneous application of heparin. The application of medical anticoagulation for cancer patients was still discussed over the last decade, even for inpatients or patients with confirmed thrombosis. The assumed higher incidence of severe bleeding events due to medical anticoagulation, especially in patients with advanced gastrointestinal malignancies, reduced the number of sufficient treated patients in the past and thus may lead to impaired overall survival $[12,19]$.

This open label phase II feasibility investigation used intensified chemotherapy and simultaneous enoxaparin treatment in patients with first line chemotherapy. The dosage of enoxaparin to be used in our trial has been intensively discussed in terms of pre-existing prevention studies in other indications. Results from the PRINCE [20] and MEDENOX [21,22] studies demonstrated effective and well-tolerated VTE prevention in patients with severe cardiopulmonary disease using the LMWH enoxaparin. The given dosages in the MEDENOX trial were set at a high prophylactic level of $40 \mathrm{mg}$ daily versus placebo and also in the PRINCE trial with $40 \mathrm{mg}$ daily versus 5000 IU unfractioned heparin $3 \times$ daily. Thus $40 \mathrm{mg}$ enoxaparin once a day was considered to be the minimal dose for primary symptomatic VTE prevention.

We used a safety step design to prevent patients from harmful increased toxicities due to the combination. The observed side effects were consistent with respect to the applied chemotherapy and were similar to those in our phase I trial [18]. We therefore continued the investigation without need of modification of the regimen. Even the addition of heparin does not lead to increased toxicities. We suspected a raise in the local bleeding rate in our advanced pancreatic cancer patients by potential cancer infiltration of the stomach or duodenum. Major bleeding rates for patients with solid cancer using warfarin were $42 \%$ and $14 \%$ for controls in 431 patients [23], in our safety trial we therefore decided to accept no higher rate than $20 \%$ using prophylactic anticoagulation with enoxaparin. Fortunately we did not observe any patient with severe bleeding under concomitant application of $40 \mathrm{mg}$ enoxaparin daily. Only one patient had a thromboembolic event while getting enoxaparin (5\%), whereas the supposed rate of thromboembolic events without heparin use would be $15-20 \%$ [11].

We further observed a slightly increased overall survival compared to gemcitabine monotherapy $[2,9,24]$. This finding and the open discussion about an independent effect 


\section{Multivariate Analysis}

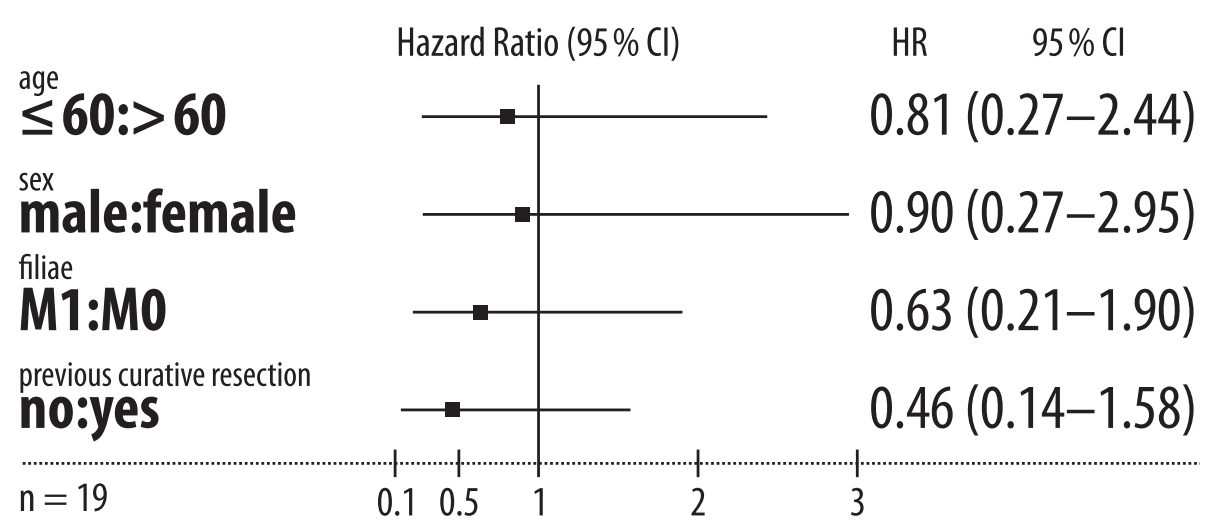

A hazard ratio below 1.0 indicates a favorable prognosis for the category mentioned last

Figure 3 Forest plot of prognostic factors.

of heparins with regard to metastasis and cancer growth has to be investigated by a randomized trial in a larger setting [17]. On the other hand this observed effect is commonly seen in phase II studies with the potential bias of unconscious patient selection and the addition of placebo effects $[7,10]$. But besides these considerations we can at least assume that the efficacy of the treatment is not negatively affected.

The finding of this investigation regarding feasibility and safety gives us confidence for using the researched combination in a larger trial to investigate the effect of heparin in a randomized setting.

\section{Conclusions}

In summary, the addition of enoxaparin to GFFC is feasible, safe and does not appear to affect the activity or the toxicity profile of the chemotherapy regimen in patients with advanced pancreatic adenocarcinoma. To examine whether enoxaparin reduces the incidence of thromboembolic events and increases survival, we have initiated a randomized investigation study of GFFC (or single-agent gemcitabine for patients with impaired performance status) with or without enoxaparin (CONKO-004) [17].

\section{Competing interests}

No potential conflicts of interest relevant to this report of our investigator initiated trial are present

\section{Acknowledgements}

This study was supported by "Charité - Forschungsförderung", the DKG (German cancer association) and the AIO ("Arbeitsgemeinschaft Internistische Onkologie"). We thank all patients for the participation and their families for their trust. Additional thanks go to the staff of the CONKO study group.

\section{Author details}

'Department of Hematology/Oncology, CharitéCentrum für Tumormedizin, Charité - Universitätsmedizin Berlin, Augustenburger Platz 1, Berlin 13353, Germany. ${ }^{2}$ Klinik für Hämatologie und Onkologie, St. Marien-Hospital, Hamm, Germany. ${ }^{3}$ Visceral- und Transplantationschirurgie, Klinik für Allgemein, Charité - Universitätsmedizin Berlin, Berlin, Germany. ${ }^{4}$ Klinik für Radiologie, Charité - Universitätsmedizin Berlin, Berlin, Germany.

Received: 3 September 2013 Accepted: 12 March 2014

Published: 19 March 2014

\section{References}

1. Jemal A, Bray F, Center MM, Ferlay J, Ward E, Forman D: Global cancer statistics. CA Cancer J Clin 2011, 61(2):69-90.

2. Burris HA 3rd, Moore MJ, Andersen J, Green MR, Rothenberg ML, Modiano MR, u. a.: Improvements in survival and clinical benefit with gemcitabine as first-line therapy for patients with advanced pancreas cancer: a randomized trial. J Clin Oncol 1997, 15(6):2403-2413.

3. Moore MJ, Hamm J, Dancey J, Eisenberg PD, Dagenais M, Fields A, u. a.: Comparison of gemcitabine versus the matrix metalloproteinase inhibitor BAY 12-9566 in patients with advanced or metastatic adenocarcinoma of the pancreas: a phase III trial of the national cancer institute of Canada clinical trials group. J Clin Oncol 2003, 21(17):3296-3302.

4. Arshad A, Al-Leswas D, Al-Taan O, Stephenson J, Metcalfe M, Steward WP u. a.: Pooled survival and response data from phase III randomized controlled trials for gemcitabine-based regimes in the treatment of advanced pancreatic cancer. Am J Clin Oncol 2013, 36(4):411-414.

5. Stathis A, Moore MJ: Advanced pancreatic carcinoma: current treatment and future challenges. Nat Rev Clin Oncol. März 2010, 7(3):163-172.

6. Pelzer U: First-line chemotherapy in advanced pancreatic cancer. Recent Results Cancer Res 2008, 177:57-60.

7. Heinemann V, Philip PA, Pelzer U: Accomplishments in 2008 in the treatment of metastatic pancreatic cancer. Gastrointest Cancer Res 2009, 3(5 Supplement 2):S43-S47.

8. Cunningham D, Chau I, Stocken DD, Valle JW, Smith D, Steward W, u. a Phase III randomized comparison of gemcitabine versus gemcitabine plus capecitabine in patients with advanced pancreatic cancer. J Clin Oncol 2009, 27(33):5513-5518. 
9. Conroy T, Desseigne F, Ychou M, Bouché O, Guimbaud R, Bécouarn Y, U. a. FOLFIRINOX versus gemcitabine for metastatic pancreatic cancer. $N$ Engl J Med 2011, 364(19):1817-1825

10. Heinemann V, Boeck S, Hinke A, Labianca R, Louvet C: Meta-analysis of randomized trials: evaluation of benefit from gemcitabine-based combination chemotherapy applied in advanced pancreatic cancer. BMC Cancer 2008, 8:82.

11. Menapace LA, Peterson DR, Berry A, Sousou T, Khorana AA: Symptomatic and incidental thromboembolism are both associated with mortality in pancreatic cancer. Thromb Haemost 2011, 106(2):371-378.

12. Sørensen HT, Mellemkjaer L, Olsen JH, Baron JA: Prognosis of cancers associated with venous thromboembolism. N Engl J Med 2000, 343(25):1846-1850.

13. Khorana AA: Cancer and thrombosis: implications of published guidelines for clinical practice. Ann Oncol 2009, 20(10):1619-1630.

14. Lee AYY, Rickles FR, Julian JA, Gent M, Baker RI, Bowden C, u. a.: Randomized comparison of low molecular weight heparin and coumarin derivatives on the survival of patients with cancer and venous thromboembolism. J Clin Oncol 2005, 23(10):2123-2129.

15. Chew HK, Davies AM, Wun T, Harvey D, Zhou H, White RH: The incidence of venous thromboembolism among patients with primary lung cancer. $J$ Thromb Haemost 2008, 6(4):601-608.

16. Icli F, Akbulut H, Utkan G, Yalcin B, Dincol D, Isikdogan A, u. a: Low molecular weight heparin (LMWH) increases the efficacy of cisplatinum plus gemcitabine combination in advanced pancreatic cancer. I Surg Oncol 2007, 95((6):507-512

17. Riess H, Pelzer U, Hilbig A, Stieler J, Opitz B, Scholten T, u. a.: Rationale and design of PROSPECT-CONKO 004: a prospective, randomized trial of simultaneous pancreatic cancer treatment with enoxaparin and chemotherapy). BMC Cancer 2008, 8:361.

18. Oettle H, Arnold D, Kern M, Hoepffner N, Settmacher U, Neuhaus P, u. a.: Phase I study of gemcitabine in combination with cisplatin, 5 -fluorouracil and folinic acid in patients with advanced esophageal cancer. Anticancer Drugs 2002, 13(8):833-838.

19. Khorana AA: Venous thromboembolism and prognosis in cancer. Thromb Res. Juni 2010, 125(6):490-493.

20. Kleber F-X, Witt C, Vogel G, Koppenhagen K, Schomaker U, Flosbach CW: Randomized comparison of enoxaparin with unfractionated heparin for the prevention of venous thromboembolism in medical patients with heart failure or severe respiratory disease. Am Heart J 2003, 145(4):614-621.

21. Turpie AG: Thrombosis prophylaxis in the acutely ill medical patient insights from the prophylaxis in MEDical patients with ENOXaparin (MEDENOX) trial. Am J Cardiol 2000, 86(12B):48M-52M.

22. Turpie AGG, Norris TM: Thromboprophylaxis in medical patients: the role of low-molecular-weight heparin. Thromb Haemost. Juli 2004, 92(1):3-12.

23. Zacharski LR, Henderson WG, Forman WB, Edwards RL, Cornell CJ Jr, Forcier RJ, u.a.: Bleeding complications from warfarin anticoagulation in patients with malignancy. J Med 1985, 16(5-6):535-561.

24. Moore MJ, Goldstein D, Hamm J, Figer A, Hecht JR, Gallinger S, U. a: Erlotinib plus gemcitabine compared with gemcitabine alone in patients with advanced pancreatic cancer: a phase III trial of the National Cancer Institute of Canada Clinical Trials Group. I Clin Oncol 2007, 25(15):1960-1966.

\section{Submit your next manuscript to BioMed Central and take full advantage of:}

- Convenient online submission

- Thorough peer review

- No space constraints or color figure charges

- Immediate publication on acceptance

- Inclusion in PubMed, CAS, Scopus and Google Scholar

- Research which is freely available for redistribution 